\title{
Identification of an inhibitor of the ubiquitin- proteasome system that induces accumulation of polyubiquitinated proteins in the absence of blocking of proteasome function
}

Cite this: Med. Chem. Commun., 2014, 5,376

\author{
Caroline Haglund, $\uparrow^{\mathrm{a}}$ Chitralekha Mohanty, $\dagger^{\mathrm{b}}$ Mårten Fryknäs, ${ }^{\mathrm{a}}$ Padraig D'Arcy, ${ }^{\mathrm{b}}$ \\ Rolf Larsson, ${ }^{a}$ Stig Linder ${ }^{\star a b}$ and Linda Rickardson ${ }^{a}$
}

The ubiquitin-proteasome system (UPS) represents one of the most promising therapeutic targets in oncology to emerge in recent years. Here we used a combination of cytotoxic and image-based screening assays to identify a novel UPS inhibitor, designated HRF-3. HRF-3 evokes a gene expression profile similar to that of other characterized UPS inhibitors, suggesting a common mechanism of action. Consistent with UPS inhibition, HRF-3 induced strong accumulation of polyubiquitinated proteins in cells. Surprisingly, HRF-3 induced only weak accumulation of two proteasome targeted reporter proteins, Ub $b^{G 76 V}$-YFP and ZsGreen-ODC. Consistent with this observation, HRF-3 did not inhibit proteasome proteolytic activity in an in vitro assay. Similar to a number of other UPS inhibitors, HRF-3 increased the expression of the redox-inducible protein Hmox-1. In distinction to the $20 \mathrm{~S}$ inhibitor bortezomib, but similarly to two different p97/VCP inhibitors, HRF-3 did not elicit strong induction of the chaperone Hsp70B'. Finally, we show that HRF-3 is cytotoxic to a variety of cancer cell lines and ex vivo

Received 17th December 2013 Accepted 28th December 2013 DOI: $10.1039 / \mathrm{c} 3 \mathrm{md} 00386 \mathrm{~h}$ www.rsc.org/medchemcomm patient tumour cells, with the strongest activity observed in cells of leukemic/myeloma origin. Taken together our data show that HRF-3 induces polyubiquitin accumulation in the absence of efficient proteasomal blocking, and suggest that induction of oxidative stress is a common denominator of UPS inhibitors.

\section{Introduction}

The ubiquitin-proteasome system (UPS) is the main pathway for protein degradation in eukaryotic cells. Ubiquitinated proteins are degraded by the $26 \mathrm{~S}$ proteasome, a large proteolytic complex composed of a $20 \mathrm{~S}$ enzymatic core capped by one or two regulatory $19 \mathrm{~S}$ complexes. ${ }^{1,2}$ The $20 \mathrm{~S}$ core particle is a cylinder-shaped complex composed of 4 stacked heptameric $\alpha$ - and $\beta$-subunits that harbor three distinct enzyme-like activities, chymotryptic-like, tryptic-like and post-glutamyl-peptidylhydrolytic-like activity, which hydrolyze target proteins based on their respective substrate preferences. ${ }^{1}$

The targeting of proteins to the proteasome for degradation is initiated by the covalent attachment of poly-ubiquitin chains, which serve as highly specific destruction signals. ${ }^{3}$ Whereas the $20 \mathrm{~S}$ proteasome functions as the molecular shredder, the $19 \mathrm{~S}$ complex functions as a selective gate keeper, only allowing

\footnotetext{
${ }^{a}$ Department of Medical Sciences (Division of Clinical Pharmacology), Uppsala University, SE-751 85 Uppsala, Sweden

${ }^{b}$ Department of Oncology-Pathology, Karolinska Institutet, SE-171 76 Stockholm, Sweden. E-mail: Stig.Linder@ki.se; Fax: +46 83390 31; Tel: +46 851772452

$\dagger$ These two authors contributed equally to this work.
}

ubiquitin-tagged proteins access to the $20 \mathrm{~S}$ proteasome for degradation. The attachment of ubiquitin is selective and requires a sequential action of E1, E2 and E3 enzymes that activate the ubiquitin molecule and catalyse its covalent attachment to target proteins. This process is highly dynamic and can be reversed by specific deubiquitination enzymes (DUBs) adding an additional mechanism of regulation. It has been suggested that several enzymes of the ubiquitination cascade are differently expressed or activated in cancer and may therefore be appropriate drug targets. ${ }^{4,5}$

Malignant cells synthesize large quantities of proteins and thus require a functioning system for degrading misfolded proteins that would otherwise accumulate and result in proteotoxicity. Consistent with this, tumour cells have been shown to be more susceptible to proteasome inhibition compared to normal cells. ${ }^{1}$ The approval of the proteasome inhibitor bortezomib (Velcade ${ }^{\circ}$ ) for treatment of multiple myeloma and mantle cell lymphoma has verified this concept. Bortezomib displays potent anti-tumour activity and is generally well-tolerated, ${ }^{6,7}$ however several problems have emerged including doselimited toxicity and acquired drug resistance which occurs in the majority of patients, suggesting a need to identify new inhibitors of the UPS. 
The UPS is believed to be highly druggable, but has been underexplored by drug developers. ${ }^{8}$ A number of structurally unrelated molecules have been shown to inhibit the proteasome (reviewed in ref. 9). Interestingly, several cytotoxic compounds such as piperlongumine, ${ }^{\mathbf{1 0 , 1 1}}$ siomycin $\mathrm{A}^{\mathbf{1 2}}$ and WP1130, ${ }^{4}$ initially described to have alternative mechanisms of action, were subsequently shown to be potent inhibitors of the UPS. Some compounds, such as physalin $\mathrm{A}^{\mathbf{1 3}}$ and piperlongumine,${ }^{10}$ were reported to inhibit the UPS at a level distinct from the $20 \mathrm{~S}$ proteasome, but the exact mechanisms of action have not been characterized.

Considering both the structural diversity of proteasome inhibitors and the subsequent reclassification of previously unknown proteasome inhibitors, we were interested in determining the prevalence of UPS inhibitors amongst panels of cytotoxic drugs. From an initial library of 10000 drug-like small molecules we identified 382 as having anti-proliferative activity. Using an image-based assay based on proteasome inhibition we identified one compound as a potential inhibitor of the UPS. This compound named HRF-3 showed interesting antineoplastic properties, which we describe here.

\section{Results}

\section{Identification of a novel UPS inhibitor}

Proteasome inhibition was determined using an image-based method to monitor the accumulation of an ornithine decarboxylase-green fluorescent fusion protein (ZsGreen-ODC) in HEK 293 cells. This fusion protein is unstable in cells due to rapid degradation by the proteasome ${ }^{14}$ From an initial screen of 382 compounds that displayed promising cytotoxic properties, a single compound was identified to induce accumulation of the reporter protein at a level $>3$ S.D. above that of untreated control cells (Fig. 1A). The structure of this compound (phosphoric acid, 2,3-dihydro-1,1-dioxido-3-thienyl diphenyl ester), here designated HRF-3, is shown in Fig. 1B. To validate the result we examined the ability of HRF-3 to block the degradation of another proteasome substrate ( $\mathrm{Ub}^{\mathrm{G} 76 \mathrm{~V}}$-YFP), expressed by MelJuSo melanoma cells. HRF-3 was found to induce the accumulation of $\mathrm{Ub}^{\mathrm{G} 76 \mathrm{~V}}$-YFP also (Fig. 1C). The fluorescent signals obtained in both of these assays were weak compared to those obtained following treatment with the $20 \mathrm{~S}$ proteasome inhibitor bortezomib (Fig. 1A and C).

\section{The gene expression response to HRF-3 is similar to that of known UPS inhibitors}

To characterize the cellular response to HRF-3, we took advantage of the Connectivity Map database (Cmap), ${ }^{15}$ a compendium of gene expression signatures generated from drug-exposed human tumour cell lines. The Cmap database contains gene expression profiles for 1309 compounds, including the gene expression pattern induced by HRF-3 (ChemBridge ID 5155877). The gene expression profile generated by HRF-3 treatment showed considerable similarity to the profile of the well characterized proteasome inhibitor MG-262, as well as those of thiostrepton, ${ }^{12} 15 \delta-\mathrm{PGJ}_{2}{ }^{16}$ and withaferin $\mathrm{A},{ }^{17}$ compounds that have been shown to act as inhibitors of the UPS (Fig. 2). We conclude that HRF-3 induces a gene expression profile similar to that observed with several UPS inhibitors.

\section{HRF-3 induces accumulation of polyubiquitinated proteins but does not block proteasome function in cells}

Inhibition of the UPS typically results in increased levels of high molecular weight polyubiquitinated proteasomal substrates in cells. We indeed found an accumulation of K48-linked polyubiquitinated proteins over time in MelJuSo cells following treatment with $5 \mu \mathrm{M}$ HRF-3 (Fig. 3A). In both MelJuSo and HCT116 cells, the extent of polyubiquitin accumulation was similar to that observed using the $20 \mathrm{~S}$ proteasome inhibitor bortezomib (Fig. 3B and C). Simultaneous exposure to HRF-3 and bortezomib did not result in further increases of polyubiquitinated proteins (Fig. 3C). The strong accumulation of polyubiquitin was surprising considering the weak signals from the proteasome substrates ZsGreen-ODC and $\mathrm{Ub}^{\mathrm{G} 76 \mathrm{~V}}-\mathrm{YFP}$ (Fig. 1A and C). We compared the accumulation of $\mathrm{Ub}^{\mathrm{G} 76 \mathrm{~V}}-\mathrm{YFP}$ in HRF-3- and bortezomib-exposed cells using western blotting. In the experiment shown in Fig. $3 \mathrm{~B}, \mathrm{Ub}^{\mathrm{G} 76 \mathrm{~V}}$-YFP could not be detected after exposure to $5 \mu \mathrm{M}$ HRF-3 whereas the levels of the reporter were strongly increased by bortezomib.

To directly address whether HRF-3 inhibits the UPS at the level of the $20 \mathrm{~S}$ proteasome, we investigated whether HRF-3 inhibits the enzymatic activity of the 20 S proteasome using SucLLVY-AMC as a substrate. No inhibitory activity was observed at $10 \mu \mathrm{M}$ and only weak inhibition was observed at $50 \mu \mathrm{M}$ (Fig. 3D) whereas, as expected, the $20 \mathrm{~S}$ inhibitor bortezomib completely inhibited enzymatic activity.

\section{Weak induction of $\mathrm{Hsp} \mathrm{CB}^{\prime}$ expression and strong induction of Hmox-1 expression by HRF-3}

We further examined the relationship between accumulation of proteasome substrates and polyubiquitinated proteins. Similar levels of K48-linked polyubiquitinated proteins were detected in cells exposed to between 5 and $100 \mu \mathrm{M}$ HRF-3 (Fig. 4A). In contrast, $\mathrm{Ub}^{\mathrm{G} 76 \mathrm{~V}}$-YFP was detected by immunoblotting at an HRF-3 concentration of $50 \mu \mathrm{M}\left(\sim 10\right.$-fold higher than the $\mathrm{IC}_{50}$ concentration) (Fig. 4A). An increase of the CDK-inhibitor p21, a known proteasome substrate, ${ }^{18}$ was observed at $20 \mu \mathrm{M}$ with stronger increase at $50 \mu \mathrm{M}$.

Chaperone induction is expected under conditions of accumulation of misfolded proteins. HRF-3 indeed induced expression of chaperone genes such as HSPA1A (Hsp70-1A), HSPA1B (Hsp70-1B) and DNAJB4 (Hsp40) (Fig. 2). Interestingly, however, HSPA6 transcripts (encoding the chaperone Hsp70B') were not induced by HRF-3. Hsp70B' is strongly induced by the proteasome inhibitor bortezomib ${ }^{19}$ and by the deubiquitinase inhibitor b-AP15, ${ }^{20}$ and is considered as a final cellular defence mechanism against proteotoxic stress. ${ }^{21}$ As shown in Fig. 4A, Hsp70B' protein levels did not increase at HRF-3 concentrations of 5-20 $\mu \mathrm{M}$, but were induced at the concentration where the proteasome was inhibited $(50 \mu \mathrm{M})$.

We compared the response to HRF-3 to that of other compounds described to inhibit the UPS (Fig. 4B). NMS859 and 
A

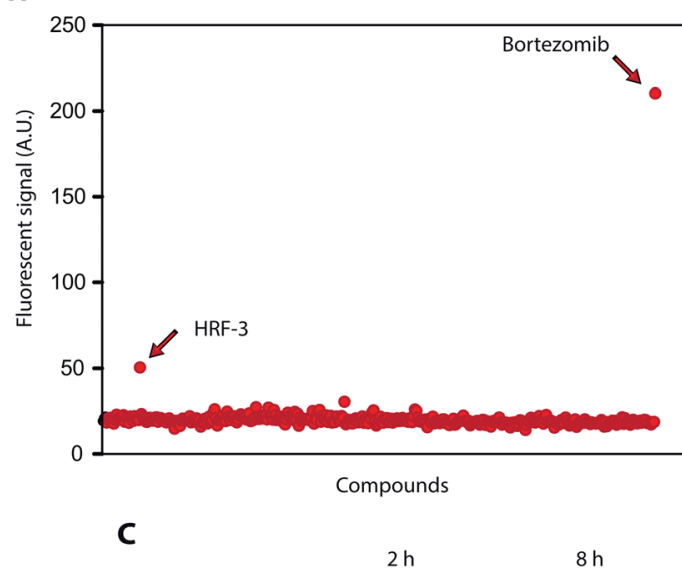

B

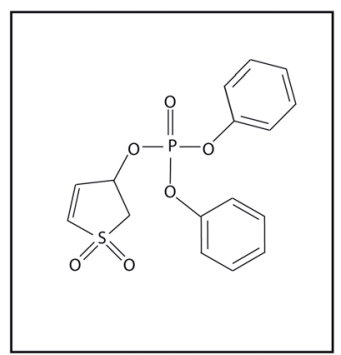

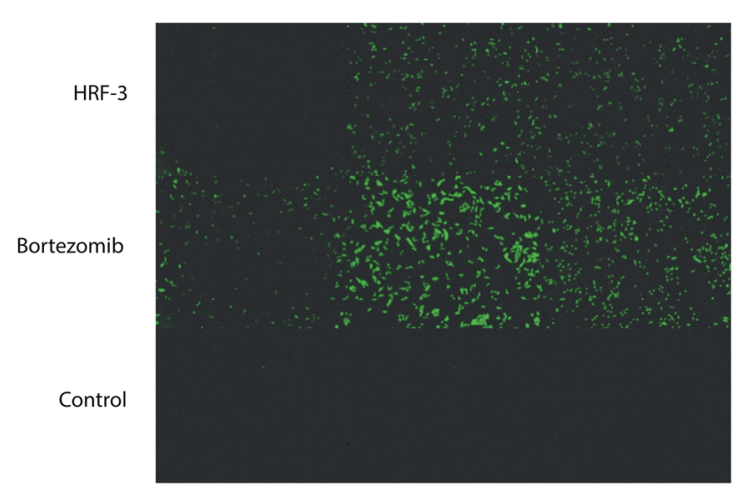

Fig. 1 Identification of HRF-3 as an UPS inhibitor. (a) Screening of 382 compounds for stabilization of ZsGreen-ODC in HEK 293 cells. Cells were exposed to $5 \mu \mathrm{M}$ of each compound for $16 \mathrm{~h}$ and the plates were analysed in an ArrayScan II instrument. HRF-3 was the only compound that generated a fluorescent signal of three standard deviations (SD) above background. Bortezomib (0.1 $\mu$ M) was used as a positive control. (b) Structural formula of HRF-3 (phosphoric acid, 2,3-dihydro-1,1-dioxido-3-thienyl diphenyl ester). (c) MelJuSo Ub ${ }^{\text {G76V }}$-YFP cells were exposed to $5 \mu \mathrm{M} \mathrm{HRF}-3$ or $0.1 \mu \mathrm{M}$ bortezomib. Live cell monitoring was performed in an IncuCyte FLR instrument.

eeyarestatin-1 (ES-1) are two recently described inhibitors of p97/VCP, an $\mathrm{AAA}(+)$ ATPase regulating endoplasmic reticulumassociated degradation. NMS859 inhibits the ATPase activity of this enzyme, ${ }^{22}$ whereas ES-1 inhibits p97/VCP-associated deubiquitinase activity. ${ }^{23}$ Piperlongumine was recently shown to inhibit the UPS independently of the activities of the $20 \mathrm{~S}$ and 19S proteasome. ${ }^{\mathbf{1 0}}$ All three compounds induced the accumulation of polyubiquitinated proteins in MelJuSo cells at concentrations of 5-10 $\mu \mathrm{M}$ (Fig. 4B) but caused little or no accumulation of the $\mathrm{Ub}^{\mathrm{G} 76 \mathrm{~V}}$-YFP reporter.

We also examined the induction of $\mathrm{Hsp} 70 \mathrm{~B}^{\prime}$ expression by these different UPS inhibitors and examined the expression of the redox-regulated marker Hmox-1. Similar to HRF-3, but distinct from bortezomib and b-AP15, p97/VCP inhibitors and piperlongumine did not induce strong $\mathrm{Hsp} 70 \mathrm{~B}^{\prime}$ expression (Fig. 4B). In contrast, all UPS inhibitors examined here elicited increases in Hmox-1 expression, the strongest increases observed with ES-1 and HRF-3 (Fig. 4B). HMOX-1 is a target gene of the redox-regulated nuclear factor-erythroid-2-related factor $2(\mathrm{Nrf}-2)^{24}$ and induction of this protein is consistent with previous reports that proteasome inhibitors induce oxidative stress. ${ }^{19,25-27}$

We conclude that HRF-3 elicits a response characterized by accumulation of polyubiquitinated proteins and increased expression of Hmox-1, whereas $\mathrm{Hsp} 70 \mathrm{~B}^{\prime}$ was not strongly induced at cytotoxic concentrations.

\section{HRF-3 is cytotoxic to cancer cell lines and primary patient tumour cells}

Since HRF-3 inhibits the UPS by a mechanism distinct from that of the clinically used agent bortezomib, we were interested in the potential activity of the drug on tumour cells. The cytotoxic activity of HRF-3 was first investigated in eight cell lines (Table 1). Myeloma cell lines are known to be highly sensitive to proteasome inhibitors. Consistent with this, the myeloma cell lines NCI-H929 and RPMI 8226 showed the highest degrees of sensitivity, with $\mathrm{IC}_{50}$ values of $0.61 \mu \mathrm{M}$ and $1.3 \mu \mathrm{M}$, respectively. The drug-resistant sub-line 8226/Dox40 and the leukemic cell lines CCRF-CEM, Kasumi-1 and HL-60 were slightly less sensitive and colon carcinoma HCT116 and MelJuSo melanoma cells were the most resistant. To investigate the anti-tumour activity of HRF-3 in a more clinically relevant setting, we treated a diverse set of primary tumour samples from patients representing colon carcinoma, pseudomyxoma peritonei (PMP), acute myeloid leukaemia (AML) and chronic lymphoblastic leukaemia (CLL) (Fig. 5). We included bortezomib as a reference UPS inhibitor. The two drugs displayed a similar pattern of 

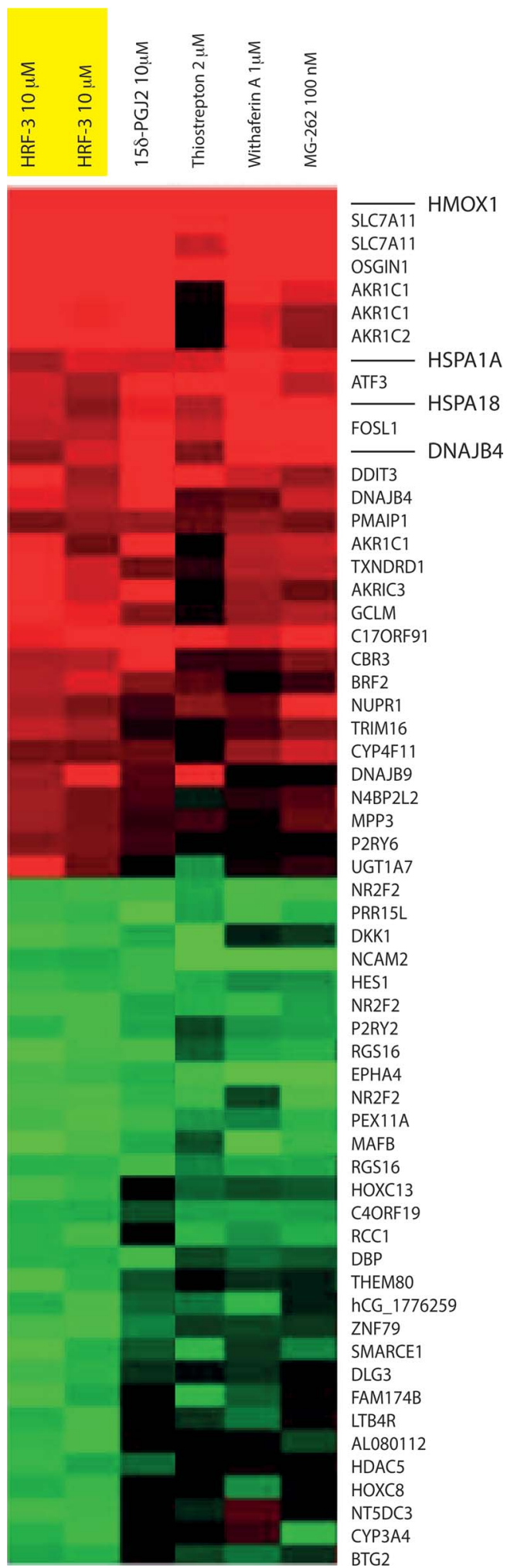

Fig. 2 The 30 most up- and down-regulated genes after treatment of MCF7 cells for $6 \mathrm{~h}$ with HRF-3. The corresponding gene expression changes after treatment with MG-262, withaferin A, thiostrepton and 15-delta prostaglandin $\mathrm{J}_{2}$ are displayed. Data are from the Cmap database. activity on the tumour types tested. CLL cells displayed the highest degree of sensitivity to $\mathrm{HRF}-3$ with a median $\mathrm{IC}_{50}$ value of $0.69 \mu \mathrm{M}$, followed by colon carcinoma samples with a median $\mathrm{IC}_{50}$ of $1.7 \mu \mathrm{M}$ (Fig. 5A). The response of pseudomyxoma peritonei (PMP) was highly variable, with $\mathrm{IC}_{50}$ values ranging from $0.51 \mu \mathrm{M}$ to $30 \mu \mathrm{M}$. A similar pattern of sensitivity was observed to bortezomib, with CLL cells being the most sensitive followed by colon carcinoma (Fig. 5B). These data suggest that HRF-3 displays anti-tumour activity on a variety of cancer types.

\section{Discussion}

The gene expression profile induced by the small molecule HRF-3 was similar to that of compounds previously shown to act as UPS inhibitors, such as MG-262, withaferin A, thiostrepton and $15 \delta-\mathrm{PGJ}_{2}$. As expected from UPS inhibition, we observed strong accumulation of polyubiquitinated proteins in tumour cells. Polyubiquitinated proteins generally accumulate as a result of decreased proteasome function, and are observed after treatment with inhibitors of $20 \mathrm{~S}$ enzymatic activity ${ }^{28}$ or $19 \mathrm{~S}$ deubiquitinase activity. ${ }^{29}$ Interestingly, at cytotoxic concentrations HRF-3 induced only weak accumulation of the ubiquitinindependent proteasome substrate ZsGreen-ODC and the ubiquitin-dependent proteasome substrate $\mathrm{Ub}^{\mathrm{G} 76 \mathrm{~V}}-\mathrm{YFP}$. Induction was, however, sufficient to be detected in an image-based screening system. In immunoblotting experiments, increased levels of Ub ${ }^{\mathrm{G} 76 \mathrm{~V}}$-YFP were detected at an HRF-3 concentration of $50 \mu \mathrm{M}$, but not at $\mathrm{IC}_{50}$ concentrations (Fig. 4A). These findings suggested that the proteasome is only partially inhibited by HRF-3 treatment at cytotoxic concentrations. This pattern is distinct from that observed with the $20 \mathrm{~S}$ proteasome inhibitor bortezomib and the 19S proteasome deubiquitinase inhibitor b-AP15 which both induce strong accumulation of $\mathrm{Ub}^{\mathrm{G} 76 \mathrm{~V}}$-YFP in cells (Fig. 4B). For both these inhibitors, accumulation of $\mathrm{Ub}^{676 \mathrm{~V}}$-YFP is strongly associated with cytotoxicity. ${ }^{27}$ These observations raise questions with regard to the origin of polyubiquitinated proteins observed in HRF-3-treated cells and why these proteins are not recognized and degraded by the proteasome.

Drug screening has identified other small molecules that inhibit the UPS at levels other than the proteasome. Piperlongumine is a natural product from the plant Piper longum (Long pepper) described to induce oxidative stress and to induce tumour cell-specific cell death. ${ }^{30} \mathrm{We}$ and others recently reported that piperlongumine is a UPS inhibitor that generates polyubiquitinated proteins in the absence of $20 \mathrm{~S}$ proteasome inhibition. ${ }^{10,11}$ We here confirmed the induction of polyubiquitinated proteins in the absence of accumulation of $\mathrm{Ub}^{\mathrm{G} 76 \mathrm{~V}}$-YFP by this compound. Physalin $\mathrm{B}$ is a steroid-like compound isolated from the plant Physalis angulata that was identified in a cell-based screen for UPS inhibitors similar to ours. ${ }^{13}$ Physalin B increases the levels of polyubiquitinated proteins but does not block the enzymatic activity of the $20 \mathrm{~S}$ proteasome. HRF-3, piperlongumine and physalin B are likely to block the UPS at step(s) upstream of the proteasome, such as endoplasmic reticulum (ER)-associated protein degradation (ERAD), ${ }^{31}$ shuttling of polyubiquitinated substrate proteins to 
A

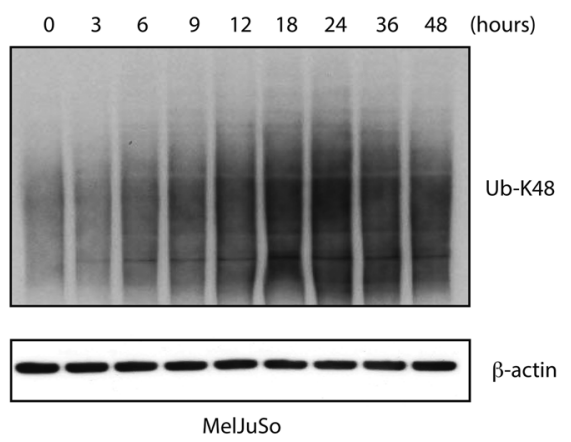

C

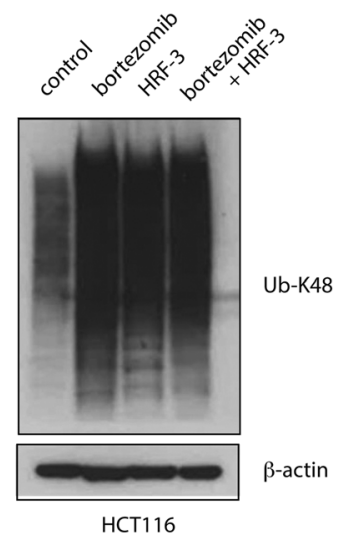

D
B

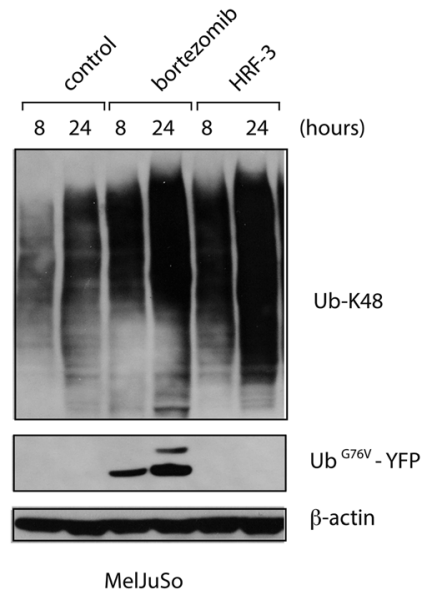

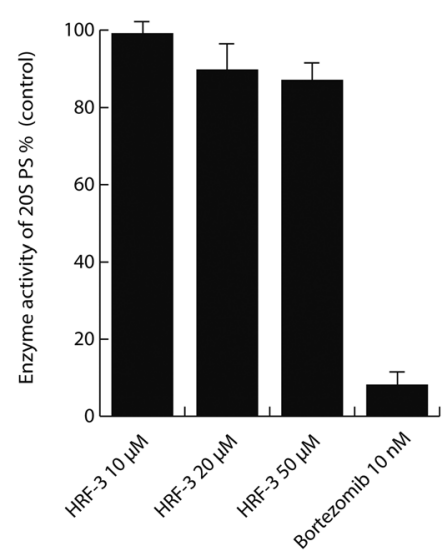

Fig. 3 HRF-3 induces accumulation of polyubiquitinated proteins but does not inhibit the $20 \mathrm{~S}$ proteasome. (a) MelJuSo Ub ${ }^{\mathrm{G} 76 \mathrm{~V}}$-YFP cells were exposed to $10 \mu \mathrm{M}$ HRF-3 for the indicated time points, harvested and subjected to immunoblotting for accumulation of K48-linked ubiquitin chains and $\beta$-actin. (b) Accumulation of polyubiquitin conjugates (Ub-K48) and Ub G76V - YFP in MelJuSo Ub ${ }^{\mathrm{G} 76 \mathrm{~V}}$-YFP cells exposed to bortezomib $(0.1 \mu \mathrm{M})$ or HRF3 $(10 \mu \mathrm{M})$ for 6 or 18 hours. (c) HCT116 cells were exposed to indicated concentrations of HRF-3 (10 $\mu \mathrm{M})$ and/or bortezomib $(0.1 \mu \mathrm{M})$ for 18 hours, harvested and subjected to immunoblotting for K48-linked ubiquitin chains and $\beta$-actin. (d) A cell free assay was used to investigate the inhibitory activity on the chymotrypsin activity on the proteasome. The enzymatic activity was measured by adding HRF-3 and bortezomib to human erythrocyte proteasome 20 S enzyme. No inhibitory activity of HRF-3 was detected under the experimental conditions. Mean values $(n=3)$ and standard deviation are displayed.

the proteasome $\mathrm{e}^{32}$ or binding of substrates to ubiquitin receptors. p97 (valosin-containing protein: p97/VCP; Cdc48p in yeast) is an $\mathrm{AAA}(+)$ ATPase that is instrumental for extraction of ERAD substrates. $^{33}$ We here examined the effects of NMS859, an inhibitor of the p97/VCP ATPase, and eeyarestatin-1, an inhibitor of p97/VCP-associated DUB activity. ${ }^{22,23}$ We show that both inhibitors induce the accumulation of polyubiquitinated substrates in a manner similar to the proteasome inhibitor bortezomib, although neither compound induces accumulation of the $\mathrm{Ub}^{\mathrm{G} 76 \mathrm{~V}}$-YFP reporter. These results show that interference with the UPS at pre-proteasomal steps give a similar response to that observed with HRF-3, piperlongumine and physalin-B. The exact mechanism(s) of action of these different orphan UPS inhibitors will be interesting to evaluate in future studies.

Stabilization of various proteasomal substrates such as $\mathrm{IkB}^{34}$ and $\mathrm{c}-\mathrm{Myc}^{35}$ have been suggested to being instrumental to proteasome inhibitor-induced cytotoxicity (reviewed in ref. 36 and 37). It is therefore interesting to note that HRF-3 as well as other UPS inhibitors such as piperlongumine, NMS859 and eeyarestatin-1 that do not appear to block proteasome degradative function are cytotoxic to tumour cells. Production of reactive oxygen species (ROS) by proteasome inhibitors has been suggested to be of importance for cytotoxicity of these agents. ${ }^{19,25,26}$ Interestingly, we observed induction of Hmox-1, a gene associated with oxidative stress, ${ }^{24}$ by all 6 agents tested (Fig. 4B). Involvement of oxidative stress therefore seems plausible with regard to the cytotoxicity of these different drugs.

As previously reported, ${ }^{19,20}$ bortezomib and b-AP15 induced strong expression of $\mathrm{Hsp} 70 \mathrm{~B}^{\prime}$, a highly inducible form of Hsp70. ${ }^{21}$ The strong accumulation of polyubiquitinated proteins by HRF-3 and compounds such as ES-1 was expected to induce expression of this chaperone, but only limited Hsp70B' induction was observed. Heat shock proteins have been described to interact directly or indirectly with the proteasome, presumably to aid proteasomal degradation. ${ }^{38}$ Chaperones are known to protect cells from proteotoxic stress ${ }^{39}$ and it is 
A

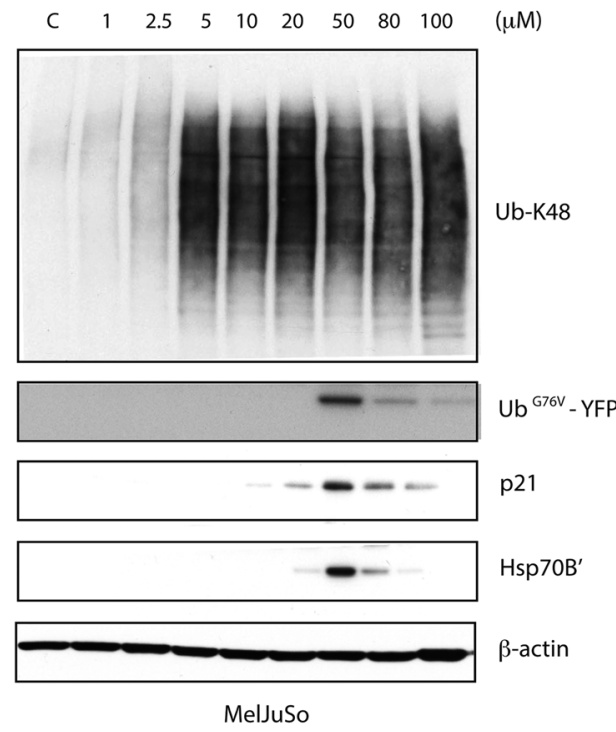

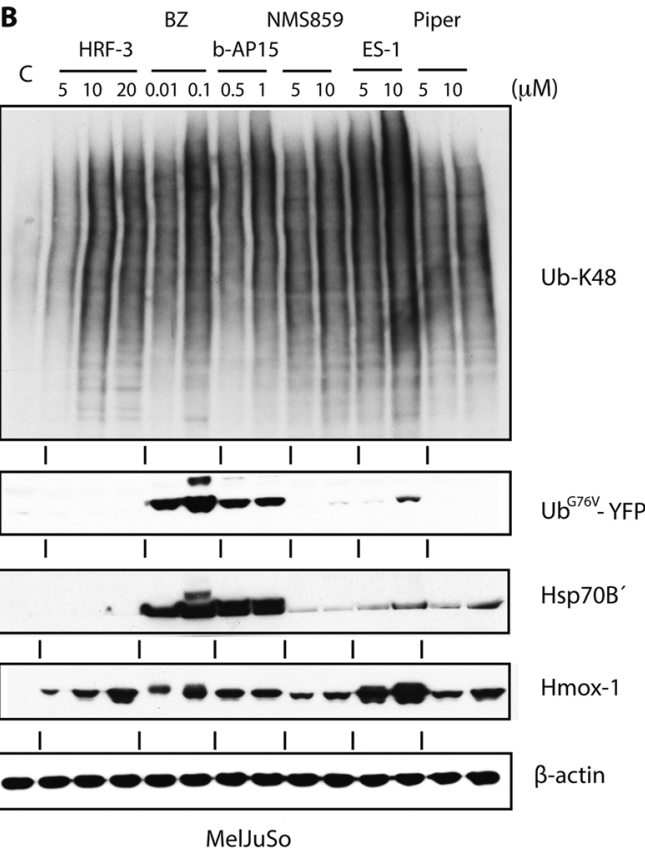

Fig. 4 Inhibition of the ubiquitin proteasome system by HRF-3. (a) MelJuSo Ub G76V HRF-3 for 18 hours, harvested and subjected to immunoblotting for accumulation of K48-linked ubiquitin chains, Ub-YFP, p21, Hsp70B' and $\beta$-actin. (b) MelJuSo Ub G76V - YFP cells were exposed to the indicated concentrations of HRF-3, bortezomib (BZ), b-AP15, NMS859, ES-1 and piperlongumine for 18 hours. Cells were harvested and subjected to immunoblotting for K48-linked ubiquitin chains, Ub-YFP, Hsp70B', Hmox-1 and $\beta$-actin.

Table 1 Summary of $\mathrm{IC}_{50}$ values in human tumour cell lines

\begin{tabular}{lll}
\hline Cell line & Origin & $\mathrm{IC}_{50} \mu \mathrm{M}(95 \% \mathrm{CI})$ \\
\hline RPMI 8226 & Myeloma & $1.3(1.1-1.5)$ \\
8226/Dox40 & Myeloma & $2.8(2.4-3.1)$ \\
NCI-H929 & Myeloma & $0.61(0.54-0.69)$ \\
CCRF-CEM & T-Cell leukaemia & $1.8(1.6-2.0)$ \\
Kasumi-1 & Acute myeloid leukaemia & $1.9(1.7-2.3)$ \\
HL-60 & Acute myeloid leukaemia & $2.5(2.3-2.8)$ \\
HCT116 & Colon carcinoma & $5.8(5.4-6.2)$ \\
MelJuSo & Melanoma & $5.4(5.1-5.8)$ \\
& &
\end{tabular}

interesting to speculate whether the lack of induction of Hsp70B' contributes to the cytotoxicity of compounds such as HRF-3 and ES-1.

When investigating the cytotoxic activity of HRF-3, myeloma cell lines and leukaemia cell lines were the most sensitive, in agreement with the known cytotoxic activity of bortezomib. ${ }^{36}$ The cytotoxic activity of HRF-3 was also investigated in patient tumour cells since it has previously been shown that in vitro activity of primary tumour samples can predict phase II activity in patients. ${ }^{\mathbf{4 0 , 4 1}}$ Compared to cell lines, the disease-specific phenotype of primary tumour cells is largely intact with respect to drug sensitivity, thus primary cells may therefore be better predictors of clinical activity. ${ }^{\mathbf{4 0}}$ Another difference is that primary tumour samples are nonproliferating and generally more drug resistant compared to highly proliferative cell lines. Surprisingly, in our study, primary tumour samples were more sensitive to HRF-3 than
A
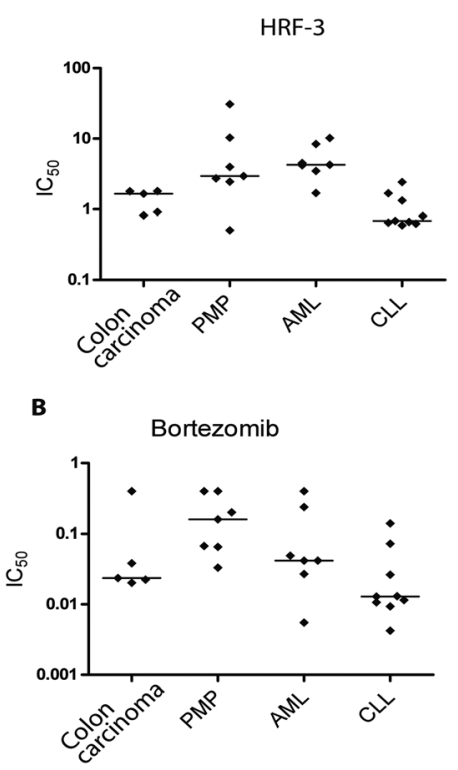

Fig. 5 Cytotoxic activity of HRF-3 (a) and bortezomib (b) in individual patient samples from colon carcinoma, pseudomyxoma peritonei (PMP), acute myelocytic leukaemia (AML) and chronic lymphatic leukaemia (CLL) expressed as $I_{50}$ in $\mu \mathrm{M}$. The median $I C_{50}$ for each diagnosis is indicated.

the cell lines. When studying the activity of bortezomib in the same patient samples as HRF-3, a similar activity profile was found. 
In conclusion, we here describe a novel inhibitor of the UPS that shows cytotoxic activities on human tumour cells. The compound was found to induce cellular polyubiquitin accumulation in the absence of efficient proteasome blocking. The exact mechanisms of action of HRF-3, as well as other UPS inhibitors such as piperlongumine and physalin $\mathrm{B}$, will be interesting to evaluate. The findings suggest that chemical biology will be a powerful tool in dissecting details on the UPS that are presently unclear.

\section{Experimental procedures}

\section{Compounds}

A chemical library consisting of 10000 drug-like small molecules was purchased from Chembridge (San Diego, CA, USA). HRF-3 (Phosphoric acid, 2,3-dihydro-1,1-dioxido-3-thienyl diphenyl ester) was purchased from Chembridge Hit2lead (San Diego, CA, USA) and bortezomib was purchased from LC Laboratories (Woburn, MA, USA). The 19S inhibitor b-AP15 was synthesized by OncoTargeting AB (Uppsala, Sweden) (also available from Boston Biochem, Cambridge, MA). The VCP/P97 inhibitor NMS859 was purchased from Xcessbio. Piperlongumine was purchased from Sigma-Aldrich (Stockholm, Sweden) and ES1 was a gift from Prof. Stephen High, University of Manchester. Dimethyl sulfoxide (DMSO) was used as the solvent for stock preparations and all further dilutions were performed in sterile water or phosphate buffered saline (PBS) with a maximum of $1 \%$ DMSO in the cell culture. For experiments, 384-well microplates (Nunclon surface, NUNC Brand Products, Roskilde, Denmark) were prepared with $5 \mu \mathrm{l}$ of respective compound in replicate at ten times the desired final drug concentrations using a Biomek 2000 pipetting station (Beckman Coulter Inc, Fullerton, CA, USA). The plates were stored at $-70{ }^{\circ} \mathrm{C}$ until further use.

\section{Cancer cell lines}

The cell lines used for cytotoxicity measurements were: the myeloma cell lines NCI-H929 and RPMI-8226, the acute myeloid leukaemia cell lines Kasumi-1 and HL-60 and the colon carcinoma cell line HCT116, all obtained from the American Type Culture collection (ATCC, Rockville, MD, USA). The 8226/Dox40 cell line, which is selected for P-gp mediated doxorubicin resistance, was a kind gift from WS Dalton, Department of Medicine, Arizona Cancer Center, University of Arizona, Tucson, AZ, USA. The T-cell leukaemia cell line CCRF-CEM was a kind gift from WT Beck, Department of Pharmacology, College of Medicine, University of Tennessee, Memphis, TN, USA. All cell lines were cultured in RPMI 1640 medium, with the exception of HCT116, which was cultured in McCoy's 5A medium. The cell plating density was 5000 cells per well for all cell lines. The cells were cultured at $37{ }^{\circ} \mathrm{C}$ in a humidified atmosphere containing $5 \% \mathrm{CO}_{2}$, and cell culturing medium supplemented with $10 \%$ heat-inactivated fetal calf serum (FCS), $2 \mathrm{mM}$ glutamine, $100 \mu \mathrm{g} \mathrm{ml}^{-1}$ streptomycin and $100 \mathrm{U} \mathrm{ml}^{-1}$ penicillin (Sigma-Aldrich, Stockholm, Sweden). The stably transfected HEK 293 ZsGreen Proteasome Sensor cell line expressing the ZsProSensor-1 protein (BD Bioscience, San Jose, CA, USA), used for screening of proteasome inhibitors, was grown in Dulbecco's Modified Eagle's medium (DMEM) supplemented as above with addition of the antibiotic G418 at $0.2 \mathrm{mg} \mathrm{ml} \mathrm{m}^{-1}$ to select stably transfected cells. The cell line MelJuSo $\mathrm{Ub}^{\mathrm{G} 76 \mathrm{~V}}$-YFP (a kind gift from Professor Nico Dantuma, Karolinska Institutet), a human melanoma cell line expressing ubiquitin fused to yellow fluorescent protein (YFP), was grown in DMEM.

\section{Measurement of cytotoxicity}

Cell survival was measured using the non-clonogenic fluorometric microculture cytotoxicity assay (FMCA), described previously in detail. ${ }^{42,43}$ The FMCA is based on the measurement of fluorescence generated from hydrolysis of fluorescein diacetate (FDA) to fluorescein by cells with intact plasma membranes. For experiments, cells were seeded in drugprepared 384-well microplates and incubated in a humidified incubator at $37{ }^{\circ} \mathrm{C} 5 \% \mathrm{CO}_{2}$ for 3 days. Columns without drugs served as controls for normal growth and cell containing media served as blanks. After incubation, medium and drugs were aspirated, the cells were washed twice with PBS, and FDA was added. After 50-70 min of incubation, the fluorescence, which is proportional to the number of attached living cells, was measured at $485 / 520 \mathrm{~nm}$ in a fluorometer (Fluostar Optima, BMG Technologies, Germany). Cell survival is presented as the Survival Index (SI), defined as the mean fluorescence in drug treated wells as a percentage of untreated wells, with blank values being subtracted. The fluorescence in the control wells had to be at least five times the mean blank value (signal/noise ratio) and a coefficient of variation (CV) of $<30 \%$ in the control wells to be defined as a positive hit. The FMCA was used for cell survival analysis throughout.

\section{Screening for cytotoxic compounds in HCT116 colon carcinoma cells}

The chemical library was screened for cytotoxic compounds using the FMCA as described above with small modifications. HCT116 colon carcinoma cells were seeded in flat-bottomed 96-well plates (Nunc) and allowed to attach, prior to drug exposure for $72 \mathrm{~h}$. Compounds were screened at a concentration of $25 \mu \mathrm{M}$ and the criterion for a hit compound was a cell survival below $35 \%$.

\section{Screening for UPS inhibitors}

The screening for UPS inhibitors was performed as in ref. 14 with minor changes, using the HEK 293 ZsGreen Proteasome Sensor Cell Line, engineered to express an ornithine decarboxylase (ODC)-fusion green-fluorescent protein that is degraded by the proteasome without the need for prior ubiquitination. The cells were plated in black optically clear bottom ViewPlates (Perkin-Elmer, Waltham, MA, USA), left to attach and then treated with $5 \mu \mathrm{M}$ of compounds for $16 \mathrm{~h}$. Then the cell nuclei were stained with Hoechst 33342 and the plates were analysed in an ArrayScan II instrument (Thermo Fisher Scientific, Waltham, MA, USA). The criterion for a hit compound was 
a generated fluorescence of three standard deviations (SD) above the background fluorescence.

\section{Connectivity map analysis}

Data from Connectivity Map (www.broadinstitute.org/cmap/) were downloaded and visualized using TMV4. ${ }^{44}$ The 30 most upand down-regulated genes after HRF-3 treatment were selected from the two instances of HRF-3-treated MCF-7 cells (the ChemBridge ID for HRF-3 is 5155877). The expression values for the selected genes after treatment with the proteasome inhibitors MG-262, $15 \delta$ prostaglandin $\mathrm{J}_{2}\left(15 \delta-\mathrm{PGJ}_{2}\right)$, thiostrepton and withaferin A were retrieved and visualized along with HRF-3 in a heat map format.

\section{Live-cell imaging of UPS-activity}

MelJuSo $\mathrm{Ub}^{\mathrm{G} 76 \mathrm{~V}}$-YFP cells were plated in black optically clear bottom ViewPlates (Perkin-Elmer) overnight and then treated with HRF-3 at $5 \mu \mathrm{M}$. Bortezomib at $10 \mu \mathrm{M}$ was used as a positive control. Treatment with UPS-inhibiting compounds leads to accumulation of YFP in the cells, and the generated fluorescence from the ubiquitin construct was continuously detected and studied in an IncuCyte FLR (Essen BioScience Inc., Ann Arbor, MI, USA).

\section{Activity assay for $20 \mathrm{~S}$ proteasome}

Quantification of the chymotrypsin activity of the 20S proteasome in the presence of HRF-3 was determined using a cell free 20S proteasome assay kit (BostonBiochem, Cambridge, MA, USA) according to the manufacturer's instructions. Briefly, 20S activity was measured by adding HRF-3 or the known $20 \mathrm{~S}$ inhibitor bortezomib to $20 \mathrm{~S}$ erythrocyte proteasomes in reaction and activation buffer (25 mM HEPES, 0.5 mM EDTA, pH 7.6 and $0.03 \%$ SDS). The compounds were incubated with $20 \mathrm{~S}$ enzyme solution for $15 \mathrm{~min}$ at $37^{\circ} \mathrm{C}$ before the substrate solution (10 $\mu \mathrm{M}$ Suc-LLVY-AMC) was added. The increase in fluorescence was measured every 3rd minute using excitation and emission wavelengths of 340 and $450 \mathrm{~nm}$, respectively. Monitoring the increase in fluorescence over time allowed quantification of the enzymatic activity.

\section{Western blot analysis}

Protein extraction from cells was performed with RIPA lysis buffer (50 mM Tris-HCL pH 7.4, $150 \mathrm{mM} \mathrm{NaCl,} 5 \mathrm{mM}$ EDTA, $0.5 \%$ Triton-X, $0.1 \%$ SDS) freshly supplemented with protease inhibitor cocktail (Sigma-Aldrich), $10 \mathrm{mM} \mathrm{N}$-ethylmaleimide (NEM; EMD Chemicals). Protein samples were collected by centrifugation at $13000 \mathrm{~g}$ for $15 \mathrm{~min}$ at $4{ }^{\circ} \mathrm{C}$ and the concentration was measured by the Bradford (Bio-Rad, Richmond, CA, USA) assay. Proteins $(10 \mu \mathrm{g})$ were denatured under reducing conditions (1\% $\beta$-mercaptoethanol) in NuPAGE sample buffer (Invitrogen, Carlsbad, CA) and subjected to gel electrophoresis, followed by transfer to polyvinylidene difluoride (PVDF; Hybond-P, Amersham, UK) membranes. Membranes were blocked for $1 \mathrm{~h}$ in 5\% non-fat dry milk (Bio-Rad) in Tris Buffer saline Tween-20 (TBS-T) at room temperature and probed with primary antibodies overnight at $4{ }^{\circ} \mathrm{C}$ and with horseradish peroxidase-labelled secondary antibodies (anti-mouse IgG and anti-rabbit IgG) for $1 \mathrm{~h}$ at room temperature. For HRP detection, an ECL chemiluminescence kit (Amersham) was used according to the manufacturer's instructions and membranes exposed to the X-ray film. Anti-UbK48 (Apu2; Millipore, Temecula, California), anti-HspA6 (Abcam), anti-heme oxygenase 1 (BD Transduction Laboratories), anti-p21 (H-164, Santa Cruz Biotechnology), anti-GFP (4B10; Cell Signalling) and anti- $\beta$ actin (Sigma-Aldrich) primary antibodies were used.

\section{Patient tumour cells}

Tumour samples were obtained by routine surgery, diagnostic biopsy or bone marrow/peripheral blood sampling approved by the ethical committee at Uppsala University (Dnr 2007/237). The samples were obtained from patients with colon carcinoma $(n=5)$, pseudomyxoma peritonei (PMP) $(n=7)$, acute myeloid leukaemia (AML) $(n=7)$ and chronic lymphatic leukaemia (CLL) $(n=9)$. PMP is a mucinous tumour within the peritoneal cavity where the primary tumour predominately is a neoplasm of the appendix, or less commonly from an ovarian tumour. Tissue from solid tumour samples was processed by mincing with scissors, and tumour cells were isolated by collagenase dispersion followed by purification using Percoll density gradient centrifugation (GE Healthcare, Uppsala, Sweden). Leukemic cells were isolated from bone marrow or peripheral blood using density gradient centrifugation in $1.007 \mathrm{~g} \mathrm{ml}^{-1}$ Ficoll-Paque (GE Healthcare). Cell viability was determined by Trypan blue exclusion, and the proportion of tumour cells $(>70 \%)$ was assessed by inspection of May-Grünwald-Giemsastained cytospin preparations. The medium used throughout was RPMI 1640 supplemented as mentioned above and the cell plating density was 5000, 20000 and 40000 cells per well for solid tumour samples, AML and CLL, respectively.

\section{Statistics}

Statistical calculations were performed with GraphPadPrism (GraphPad Software, Inc., San Diego, CA, USA). Dose-response data were processed using log-linear interpolation to obtain log $\mathrm{IC}_{50}$ values (the concentration resulting in 50\% survival compared with control). IC $_{50}$ values are presented as the mean $\mathrm{IC}_{50}$ in the repeated experiments, with $95 \%$ confidence intervals. In cases where an $\mathrm{IC}_{50}$ could not be determined, the $\mathrm{IC}_{50}$ value was reported as the highest or lowest concentration tested. For patient samples, the median $\mathrm{IC}_{50}$ values are used throughout.

\section{Acknowledgements}

The skilful technical assistance of Kristin Blom, Anna-Karin Lannergård, Lena Lenhammar and Annika Jonasson is gratefully acknowledged. We thank Stephen High, University of Manchester, for the gift of ES-1. This work was supported by Cancerfonden, Radiumhemmets forskningsfonder, Vetenskapsrådet and Strategiska Forskningsstiftelsen. 


\section{References}

1 J. Adams, The proteasome: a suitable antineoplastic target, Nat. Rev. Cancer, 2004, 4, 349-360.

2 J. M. Peters, Z. Cejka, J. R. Harris, J. A. Kleinschmidt and W. Baumeister, Structural features of the $26 \mathrm{~S}$ proteasome complex, J. Mol. Biol., 1993, 234, 932-937.

3 T. Ravid and M. Hochstrasser, Diversity of degradation signals in the ubiquitin-proteasome system, Nat. Rev. Mol. Cell Biol., 2008, 9, 679-690.

4 V. Kapuria, L. F. Peterson, D. Fang, W. G. Bornmann, M. Talpaz and N. J. Donato, Deubiquitinase inhibition by small-molecule WP1130 triggers aggresome formation and tumor cell apoptosis, Cancer Res., 2010, 70, 9265-9276.

5 L. Bedford, J. Lowe, L. R. Dick, R. J. Mayer and J. E. Brownell, Ubiquitin-like protein conjugation and the ubiquitinproteasome system as drug targets, Nat. Rev. Drug Discovery, 2011, 10, 29-46.

6 P. G. Richardson, B. Barlogie, J. Berenson, S. Singhal, S. Jagannath, D. Irwin, S. V. Rajkumar, G. Srkalovic, M. Alsina, R. Alexanian, D. Siegel, R. Z. Orlowski, D. Kuter, S. A. Limentani, S. Lee, T. Hideshima, D. L. Esseltine, M. Kauffman, J. Adams, D. P. Schenkein and K. C. Anderson, A phase 2 study of bortezomib in relapsed, refractory myeloma, N. Engl. J. Med., 2003, 348, 2609-2617.

7 R. I. Fisher, S. H. Bernstein, B. S. Kahl, B. Djulbegovic, M. J. Robertson, S. de Vos, E. Epner, A. Krishnan, J. P. Leonard, S. Lonial, E. A. Stadtmauer, O. A. O'Connor, H. Shi, A. L. Boral and A. Goy, Multicenter phase II study of bortezomib in patients with relapsed or refractory mantle cell lymphoma, J. Clin. Oncol., 2006, 24, 4867-4874.

$8 \mathrm{P}$. Cohen and M. Tcherpakov, Will the ubiquitin system furnish as many drug targets as protein kinases?, Cell, 2010, 143, 686-693.

9 G. de Bettignies and O. Coux, Proteasome inhibitors: dozens of molecules and still counting, Biochimie, 2010, 92, 1530-1545.

10 M. Jarvius, M. Fryknas, P. D'Arcy, C. Sun, L. Rickardson, J. Gullbo, C. Haglund, P. Nygren, S. Linder and R. Larsson, Piperlongumine induces inhibition of the ubiquitinproteasome system in cancer cells, Biochem. Biophys. Res. Commun., 2013, 431, 117-123.

11 M. Halasi, M. Wang, T. S. Chavan, V. Gaponenko, N. Hay and A. L. Gartel, ROS inhibitor $N$-acetyl-L-cysteine antagonizes the activity of proteasome inhibitors, Biochem. J., 2013, 454, 201-208.

12 U. G. Bhat, M. Halasi and A. L. Gartel, FoxM1 is a general target for proteasome inhibitors, PLoS One, 2009, 4, e6593.

13 I. Vandenberghe, L. Creancier, S. Vispe, J. P. Annereau, J. M. Barret, I. Pouny, A. Samson, Y. Aussagues, G. Massiot, F. Ausseil, C. Bailly and A. Kruczynski, Physalin B, a novel inhibitor of the ubiquitin-proteasome pathway, triggers NOXA-associated apoptosis, Biochem. Pharmacol., 2008, 76, 453-462.

14 L. Rickardson, M. Wickstrom, R. Larsson and H. Lovborg, Image-based screening for the identification of novel proteasome inhibitors, J. Biomol. Screening, 2007, 12, 203-210.
15 J. Lamb, E. D. Crawford, D. Peck, J. W. Modell, I. C. Blat, M. J. Wrobel, J. Lerner, J. P. Brunet, A. Subramanian, K. N. Ross, M. Reich, H. Hieronymus, G. Wei, S. A. Armstrong, S. J. Haggarty, P. A. Clemons, R. Wei, S. A. Carr, E. S. Lander and T. R. Golub, The connectivity map: using gene-expression signatures to connect small molecules, genes, and disease, Science, 2006, 313, 19291935.

16 T. Shibata, T. Yamada, M. Kondo, N. Tanahashi, K. Tanaka, H. Nakamura, H. Masutani, J. Yodoi and K. Uchida, An endogenous electrophile that modulates the regulatory mechanism of protein turnover: inhibitory effects of 15deoxy-delta 12,14-prostaglandin J2 on proteasome, Biochemistry, 2003, 42, 13960-13968.

17 H. Yang, G. Shi and Q. P. Dou, The tumor proteasome is a primary target for the natural anticancer compound withaferin A isolated from Indian winter cherry, Mol. Pharmacol., 2007, 71, 426-437.

18 J. Bloom, V. Amador, F. Bartolini, G. DeMartino and M. Pagano, Proteasome-mediated degradation of p21 via $N$-terminal ubiquitinylation, Cell, 2003, 115, 71-82.

19 A. Fribley, Q. Zeng and C. Y. Wang, Proteasome inhibitor PS341 induces apoptosis through induction of endoplasmic reticulum stress-reactive oxygen species in head and neck squamous cell carcinoma cells, Mol. Cell. Biol., 2004, 24, 9695-9704.

20 S. Brnjic, M. Mazurkiewicz, M. Fryknas, C. Sun, X. Zhang, R. Larsson, P. D'Arcy and S. T. Linder, Induction of tumor cell apoptosis by a proteasome deubiquitinase inhibitor is associated with oxidative stress, Antioxid. Redox Signaling, 2013, PMID 24011031.

21 E. J. Noonan, R. F. Place, C. Giardina and L. E. Hightower, Hsp70B' regulation and function, Cell Stress Chaperones, 2007, 12, 393-402.

22 P. Magnaghi, R. D'Alessio, B. Valsasina, N. Avanzi, S. Rizzi, D. Asa, F. Gasparri, L. Cozzi, U. Cucchi, C. Orrenius, P. Polucci, D. Ballinari, C. Perrera, A. Leone, G. Cervi, E. Casale, Y. Xiao, C. Wong, D. J. Anderson, A. Galvani, D. Donati, T. O'Brien, P. K. Jackson and A. Isacchi, Covalent and allosteric inhibitors of the ATPase VCP/p97 induce cancer cell death, Nat. Chem. Biol., 2013, 9, 548-556.

23 Q. Wang, L. Li and Y. Ye, Inhibition of p97-dependent protein degradation by eeyarestatin I, J. Biol. Chem., 2008, 283, 7445-7454.

24 D. Morse and A. M. Choi, Heme oxygenase-1: from bench to bedside, Am. J. Respir. Crit. Care Med., 2005, 172, 660-670.

25 Y. H. Ling, L. Liebes, Y. Zou and R. Perez-Soler, Reactive oxygen species generation and mitochondrial dysfunction in the apoptotic response to bortezomib, a novel proteasome inhibitor, in human $\mathrm{H} 460$ non-small cell lung cancer cells, J. Biol. Chem., 2003, 278, 33714-33723.

26 P. Perez-Galan, G. Roue, N. Villamor, E. Montserrat, E. Campo and D. Colomer, The proteasome inhibitor bortezomib induces apoptosis in mantle-cell lymphoma through generation of ROS and Noxa activation independent of p53 status, Blood, 2006, 107, 257-264. 
27 S. Brnjic, M. Mazurkiewicz, M. Fryknas, C. Sun, X. Zhang, R. Larsson, P. D'Arcy and S. Linder, Induction of Tumor Cell Apoptosis by a Proteasome Deubiquitinase Inhibitor is Associated with Oxidative Stress, Antioxid. Redox Signaling, 2013, PMID 24011031.

28 K. T. Bush, A. L. Goldberg and S. K. Nigam, Proteasome inhibition leads to a heat-shock response, induction of endoplasmic reticulum chaperones, and thermotolerance, J. Biol. Chem., 1997, 272, 9086-9092.

29 P. D'Arcy, S. Brnjic, M. H. Olofsson, M. Fryknas, K. Lindsten, M. De Cesare, P. Perego, B. Sadeghi, M. Hassan, R. Larsson and S. Linder, Inhibition of proteasome deubiquitinating activity as a new cancer therapy, Nat. Med., 2011, 17, 1636-1640.

30 L. Raj, T. Ide, A. U. Gurkar, M. Foley, M. Schenone, X. Li, N. J. Tolliday, T. R. Golub, S. A. Carr, A. F. Shamji, A. M. Stern, A. Mandinova, S. L. Schreiber and S. W. Lee, Selective killing of cancer cells by a small molecule targeting the stress response to ROS, Nature, 2011, 475, 231-234.

31 R. K. Plemper and D. H. Wolf, Retrograde protein translocation: ERADication of secretory proteins in health and disease, Trends Biochem. Sci., 1999, 24, 266-270.

32 L. Madsen, A. Schulze, M. Seeger and R. Hartmann-Petersen, Ubiquitin domain proteins in disease, BMC Biochem., 2007, $\mathbf{1}($ suppl 8), S1.

33 D. H. Wolf and A. Stolz, The Cdc48 machine in endoplasmatic reticulum associated protein degradation, Biochim. Biophys. Acta, 2012, 1823, 117-124.

34 T. Hideshima, D. Chauhan, P. Richardson, C. Mitsiades, N. Mitsiades, T. Hayashi, N. Munshi, L. Dang, A. Castro, V. Palombella, J. Adams and K. C. Anderson, NF-kappa B as a therapeutic target in multiple myeloma, J. Biol. Chem., 2002, 277, 16639-16647.

35 M. A. Nikiforov, M. Riblett, W. H. Tang, V. Gratchouck, D. Zhuang, Y. Fernandez, M. Verhaegen, S. Varambally, A. M. Chinnaiyan, A. J. Jakubowiak and M. S. Soengas, Tumor cell-selective regulation of NOXA by c-MYC in response to proteasome inhibition, Proc. Natl. Acad. Sci. U. S. A., 2007, 104, 19488-19493.
36 T. Caravita, P. de Fabritiis, A. Palumbo, S. Amadori and M. Boccadoro, Bortezomib: efficacy comparisons in solid tumors and hematologic malignancies, Nat. Clin. Pract. Oncol., 2006, 3, 374-387.

37 D. J. McConkey and K. Zhu, Mechanisms of proteasome inhibitor action and resistance in cancer, Drug Resist. Updates, 2008, 11, 164-179.

38 D. Lanneau, G. Wettstein, P. Bonniaud and C. Garrido, Heat shock proteins: cell protection through protein triage, $\mathrm{Sci}$. World J., 2010, 10, 1543-1552.

39 A. Samali, C. I. Holmberg, L. Sistonen and S. Orrenius, Thermotolerance and cell death are distinct cellular responses to stress: dependence on heat shock proteins, FEBS Lett., 1999, 461, 306-310.

$40 \mathrm{H}$. Fridborg, E. Jonsson, P. Nygren and R. Larsson, Relationship between diagnosis-specific activity of cytotoxic drugs in fresh human tumour cells ex vivo and in the clinic, Eur. J. Cancer, 1999, 35, 424-432.

41 C. Haglund, A. Aleskog, P. Nygren, J. Gullbo, M. Hoglund, M. Wickstrom, R. Larsson and E. Lindhagen, In vitro evaluation of clinical activity and toxicity of anticancer drugs using tumor cells from patients and cells representing normal tissues, Cancer Chemother. Pharmacol., 2012, 69, 697-707.

42 R. Larsson, J. Kristensen, C. Sandberg and P. Nygren, Laboratory determination of chemotherapeutic drug resistance in tumor cells from patients with leukemia, using a fluorometric microculture cytotoxicity assay (FMCA), Int. J. Cancer, 1992, 50, 177-185.

43 E. Lindhagen, P. Nygren and R. Larsson, The fluorometric microculture cytotoxicity assay, Nat. Protoc., 2008, 3, 13641369.

44 A. I. Saeed, V. Sharov, J. White, J. Li, W. Liang, N. Bhagabati, J. Braisted, M. Klapa, T. Currier, M. Thiagarajan, A. Sturn, M. Snuffin, A. Rezantsev, D. Popov, A. Ryltsov, E. Kostukovich, I. Borisovsky, Z. Liu, A. Vinsavich, V. Trush and J. Quackenbush, TM4: a free, open-source system for microarray data management and analysis, BioTechniques, 2003, 34, 374-378. 